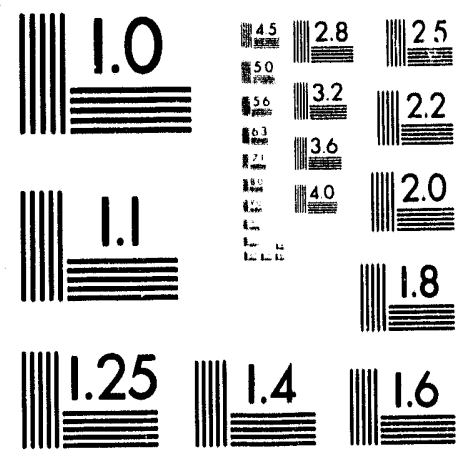



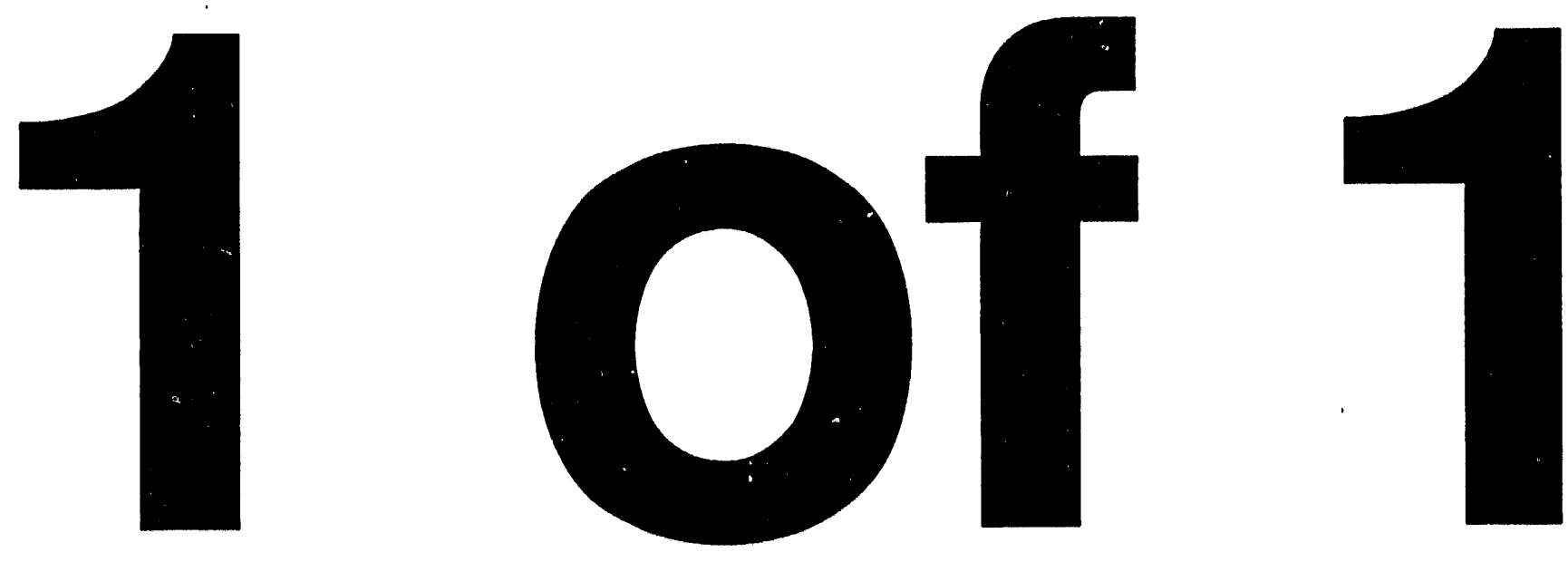


\title{
Swelling Equilibria for Cationic 2-Hydroxyethyl Methacrylate (HEMA)-Based Hydrogels
}

John P. Baker, Harvey W. Blanch and John M. Prausnitz

\author{
Department of Chemical Engineering \\ University of California
}

\author{
and \\ Chemical Sciences Division \\ Lawrence Berkeley Laboratory \\ University of California \\ Berkeley, CA 94720
}

August 1993

This work was supported by the Director, Office of Energy Research, Office of Basic Energy Sciences, Chemical Sciences Division of the U.S. Department of Energy under Contract Number DE-AC03-76SF00098. Additional support was provided by the National Institutes of Health under Grant Number R01 GM46788-01. 


\title{
Swelling Equilibria for Cationic 2-Hydroxyethyl Methacrylate
} (HEMA)-Based Hydrogels

\author{
John P. Baker, Harvey W. Blanch and John M. Prausnitz* \\ Department of Chemical Engineering, University of California, and Chemical \\ Sciences Division, Lawrence Berkeley Laboratory, 1 Cyclotron Road, Berkeley, \\ CA 94720.
}

This work was supported by the Director, Office of Energy Research, Office of Basic Energy Sciences, Chemical Sciences Division of the U.S. Department of Energy under Contract No. DE-AC03-76SF00098.

*To whom correspondence should be addressed. 


\section{Summary}

Cationic HEMA-based hydrogels were synthesized by copolymerizing HEMA with [(methacrylamido)propyl] trimethylammonium chloride (MAPTAC). Swelling equilibria were measured in pure water and in aqueous sodium chloride solutions. Hydrogel swelling is an increasing function of the MAPTAC content. A Flory-type swelling model using a concentration-dependent Flory $\chi$ parameter semi-quantitatively describes poly(HEMA co-MAPTAC) hydrogel swelling in aqueous sodium chloride.

\section{Experimental Section}

Materials. 2-Hydroxyethyl methacrylate (HEMA) and ethylene glycol dimethacrylate (EGDMA) were purchased from Polysciences. [(Methacrylamido)propyl]trimethylammonium chloride (MAPTAC) (50\% solution in water) was purchased from Aldrich. Ammonium persulfate (APS) (certified ACS grade) was purchased from Fisher. All reagents were used as received. Water was distilled, then filtered and deionized with a Barnstead Nanopure II unit.

Synthesis. Clear, homogeneous HEMA-based hydrogels with cationic character were synthesized by the free-radical copolymerization of HEMA, MAPTAC and the crosslinking monomer EGDMA. APS was used to initiate polymerization; an initiator concentration of $1 \mathrm{mg} / \mathrm{ml}$ was used in the synthesis. Water served as the diluent in the synthesis.

A series of hydrogels was prepared such that the mole percent crosslinker on a diluentfree basis $(\% C)$ and the total initial monomer concentration $(w / v)(\% T)$ in the reaction mixture were fixed at 1.0 and 65 , respectively. The mole percent MAPTAC in the reaction mixture on a diluent-free basis (\%MAPTAC) was varied from zero to 6 . The reaction mixture was poured into $10 \times 75 \mathrm{~mm}$ Pyrex test tubes. The test tubes were immersed in a water bath at $50^{\circ} \mathrm{C}$ for 24 hours. The hydrogels were liberated from the test tubes, sliced 
into $3 \mathrm{~mm}$-thick disks, then soaked in an excess of water to extract the soluble fraction and initiator residues.

Swelling. Equilibrium-swelling measurements were conducted in water and in aqueous $\mathrm{NaCl}$ solutions. We report the swelling ratio, which is the mass ratio of swollen hydrogel to dry hydrogel (Tables 1 and 2).

\section{Swelling Theory}

Measured swelling equilibria are compared with predictions from a Flory-type swelling model. The The Flory-type swelling model used for this work contains terms for polymer/solvent mixing (Flory-Huggins theory), network elasticity (phantom theory) and ion/solvent mixing (ideal Donnan equilibria). The swelling model is discussed in detail elsewhere (Baker, 1993; Baker et al., 1993a,b).

\section{Results and Discussion}

Figure 1 shows swelling equilibria in pure water for the \%MAPTAC-varying poly(HEMA co-MAPTAC) hydrogels. Swelling increases with rising \%MAPTAC. The fixed-cationic charges on the network are accompanied by osmotically-active counterions. Since the water contains no added ions, the unequal distribution of mobile ions between the hydrogel and the water provides for a net osmotic pressure which causes water to enter the hydrogel.

The nominal values of the hydrogel-preparation parameters (65\%T, $1.0 \% C, 0-6$ \%MAPTAC) and the measured swelling ratios in water were inserted into the Flory-type swelling model; Flory $\chi$ parameters were fit to the data. Table 1 provides the results of the fits. As expected, the $\chi$ parameter decreases with rising \%MAPTAC indicating that the network polymer is rendered more hydrophilic as its charge density increases. A best-fit exponential function was derived for $\chi$ as a function of swelling ratio for use in calculations 
described below. For the uncharged hydrogel, we calculated a value of 0.82 for $\chi$; other workers have obtained similar values (Peppas and Moynihan, 1987).

Figure 2 shows swelling equilibria in aqueous sodium chloride for the \%MAPTACvarying hydrogels. Hydrogel swelling declines with rising $\mathrm{NaCl}$ concentration. The added $\mathrm{NaCl}$ renders more equal the distribution of osmotically-active mobile ions between hydrogel and external solution. This levelling of the distribution of mobile ions removes the main driving force for swelling; thus swelling declines. Also shown in Figure 2 are predictions from the Flory-type swelling model including the concentration-dependent Flory $\chi$ parameter. Fair agreement between theory and experiment is obtained.

\section{Acknowledgments}

This work was supported by the Director, Office of Energy Research, Office of Basic Energy Sciences, Chemical Sciences Division of the U.S. Department of Energy under Contract Number DE-AC03-76SF00098. Additional support was provided by the National Institutes of Health under Grant Number R01 GM46788-01.

\section{References}

Baker, J. P. Equilibrium Swelling Properties of Ionized Hydrogels, Ph. D. Thesis, University of California at Berkeley, 1.993.

Baker, J. P.; Hong, L. H.; Blanch, H. W.; Prausnitz, J. M. "Effect of Initial Total Monomer Concentration on the Swelling Behavior of Cationic Acrylamide-Based Hydrogels," Macromolecules, submitted, 1993a.

Baker, J. P.; Hong, L. H.; Blanch, H. W.; Prausnitz, J. M. "Effect of Initial Total Monomer Concentration on the Swelling Behavior of Cationic Acrylamide-Based Hydrogels," LBL Report No. 34418, Lawrence Berkeley Laboratory: Berkeley, CA, $1993 b$. 
Peppas, N. A.; Moynihan, H. J. "Structure and Physical Properties of Poly(2Hydroxyethyl Methacrylate) Hydrogels," in: Hydrogels in Medicine and Pharmacy, Volume II: Polymers; Peppas, N. A., Ed.; CRC Press: Boca Raton, FL, 1987, pp. 49 $-64$. 


\section{Figures}

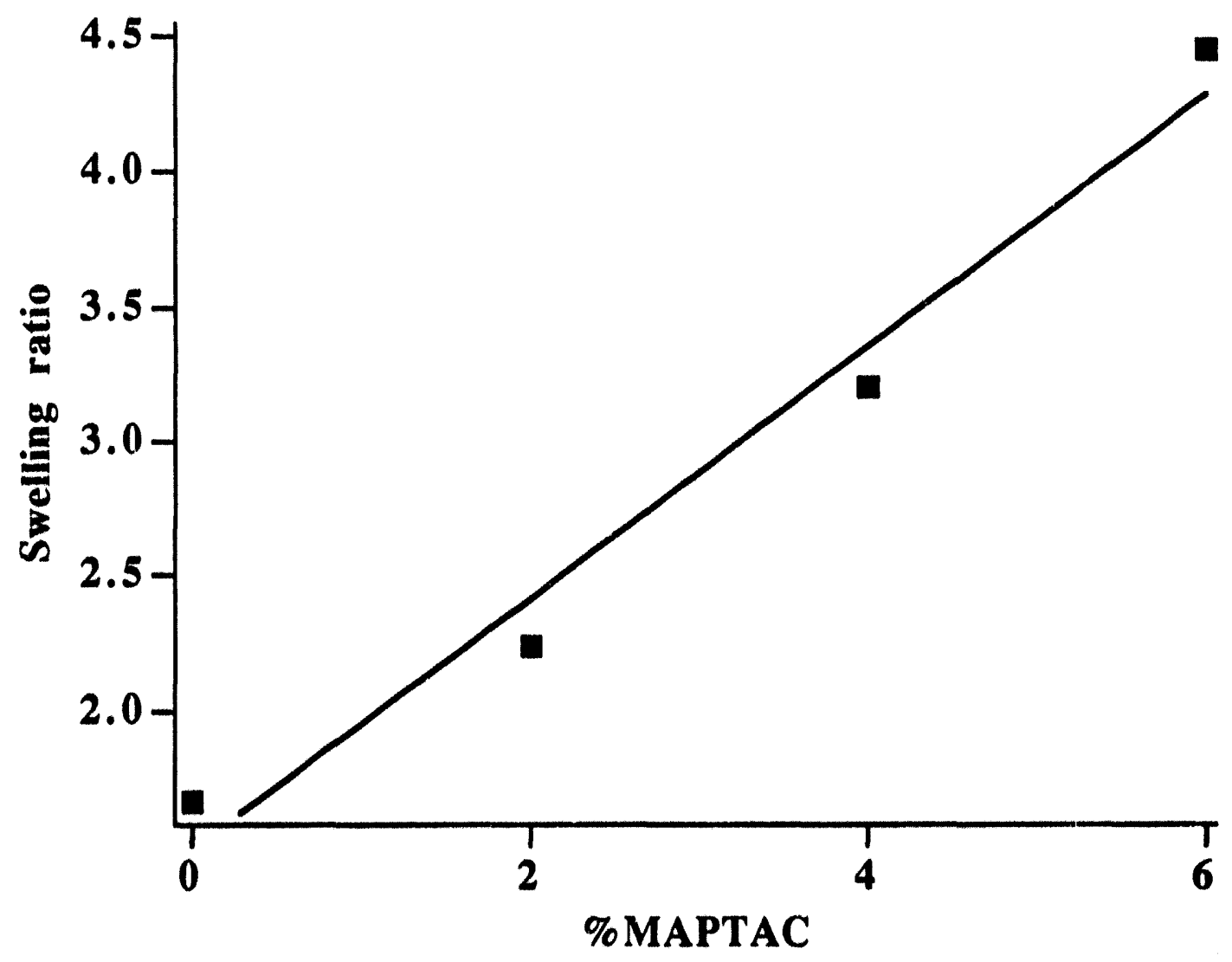

Figure 1. Swelling ratios ( $g$ swollen gel/g dry gel) in water for poly(HEMA coMAPTAC) hydrogels prepared with varying $\% M A P T A C$. The hydrogels were $65 \% T$ and $1.0 \% C$. 


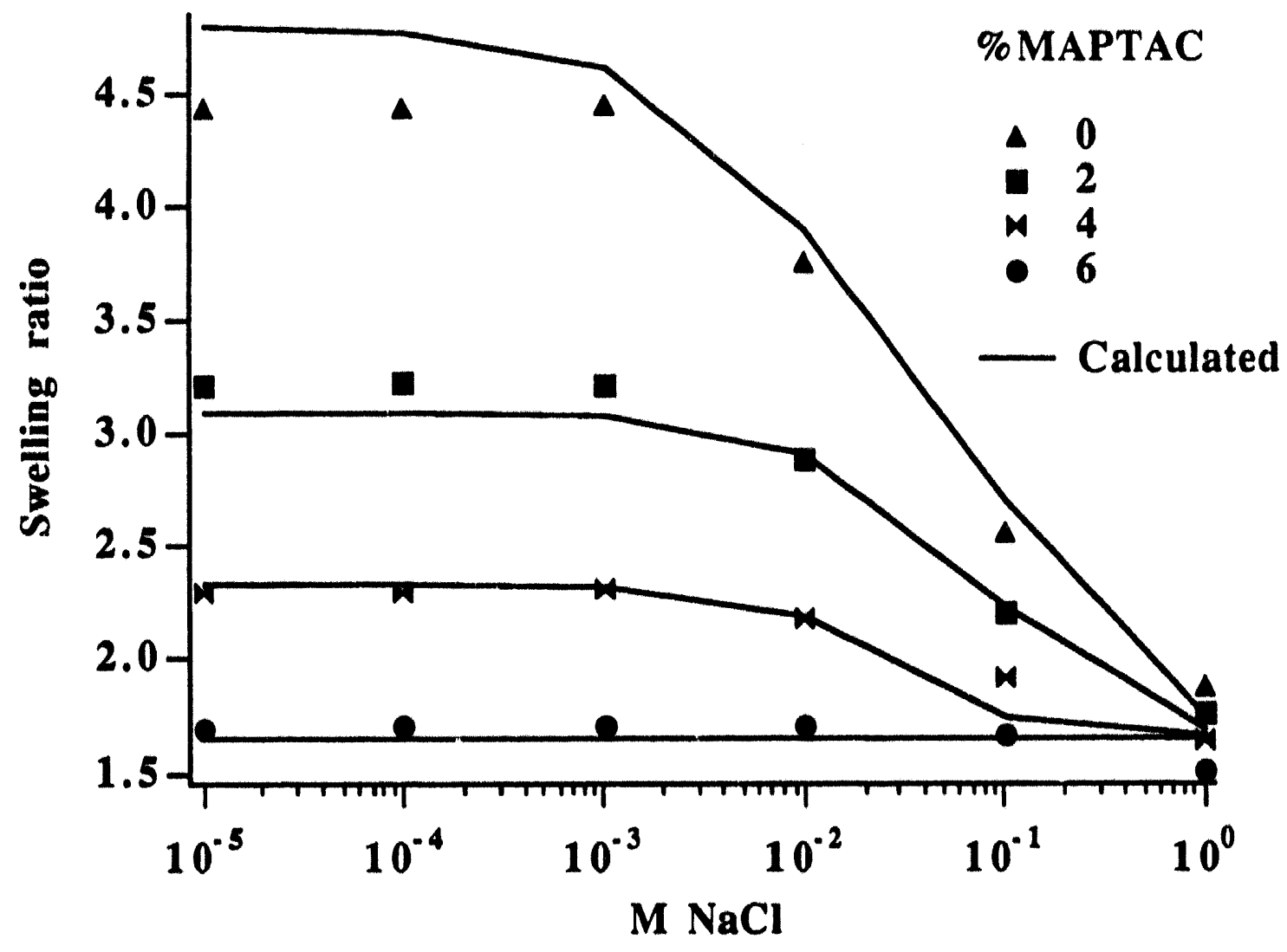

Figure 2. Comparison of measured and predicted swelling equilibria in aqueous $\mathrm{NaCl}$ for \%MAPTAC-varying poly(HEMA co-MAPTAC) hydrogels. 


\section{Tables}

Table 1. Swelling ratios in pure water for poly(HEMA co-MAPTAC) hydrogels. Also listed are Flory $\chi$ parameters fitted to the swelling data.

\begin{tabular}{c|cc}
\hline & \multicolumn{2}{|c}{ Swelling } \\
\%MAPTAC & ratio $^{\mathrm{a}}$ & $\chi$ \\
\hline 0 & 1.66 & 0.816 \\
2 & 2.24 & 0.694 \\
4 & 3.20 & 0.638 \\
6 & 4.44 & 0.633 \\
\hline
\end{tabular}

aswelling ratio $=(\mathrm{g}$ swollen $\mathrm{gel}) /(\mathrm{g}$ dry gel $)$ 
Table 2. Swelling ratios ${ }^{\mathrm{a}}$ in aqueous $\mathrm{NaCl}$ for \%MAPTAC-varying poly(HEMA coMAPTAC) hydrogels.

\begin{tabular}{l|cccc}
\hline \multirow{2}{*}{$\mathrm{M} \mathrm{NaCl}$} & 0 & 2 & 4 & 6 \\
\hline 1.0 & 1.5077 & 1.6383 & 1.7554 & 1.872 \\
0.10 & 1.6598 & 1.9106 & 2.2057 & 2.5507 \\
$1.0 \mathrm{E}-2$ & 1.7025 & 2.1713 & 2.8831 & 3.7535 \\
$1.0 \mathrm{E}-3$ & 1.7041 & 2.3094 & 3.2174 & 4.4387 \\
$1.0 \mathrm{E}-4$ & 1.7072 & 2.2899 & 3.2283 & 4.4268 \\
$1.0 \mathrm{E}-05$ & 1.6887 & 2.2914 & 3.2172 & 4.4325 \\
\hline
\end{tabular}

aSwelling ratio $=(\mathrm{g}$ swollen gel $) /(\mathrm{g}$ dry gel $)$ 

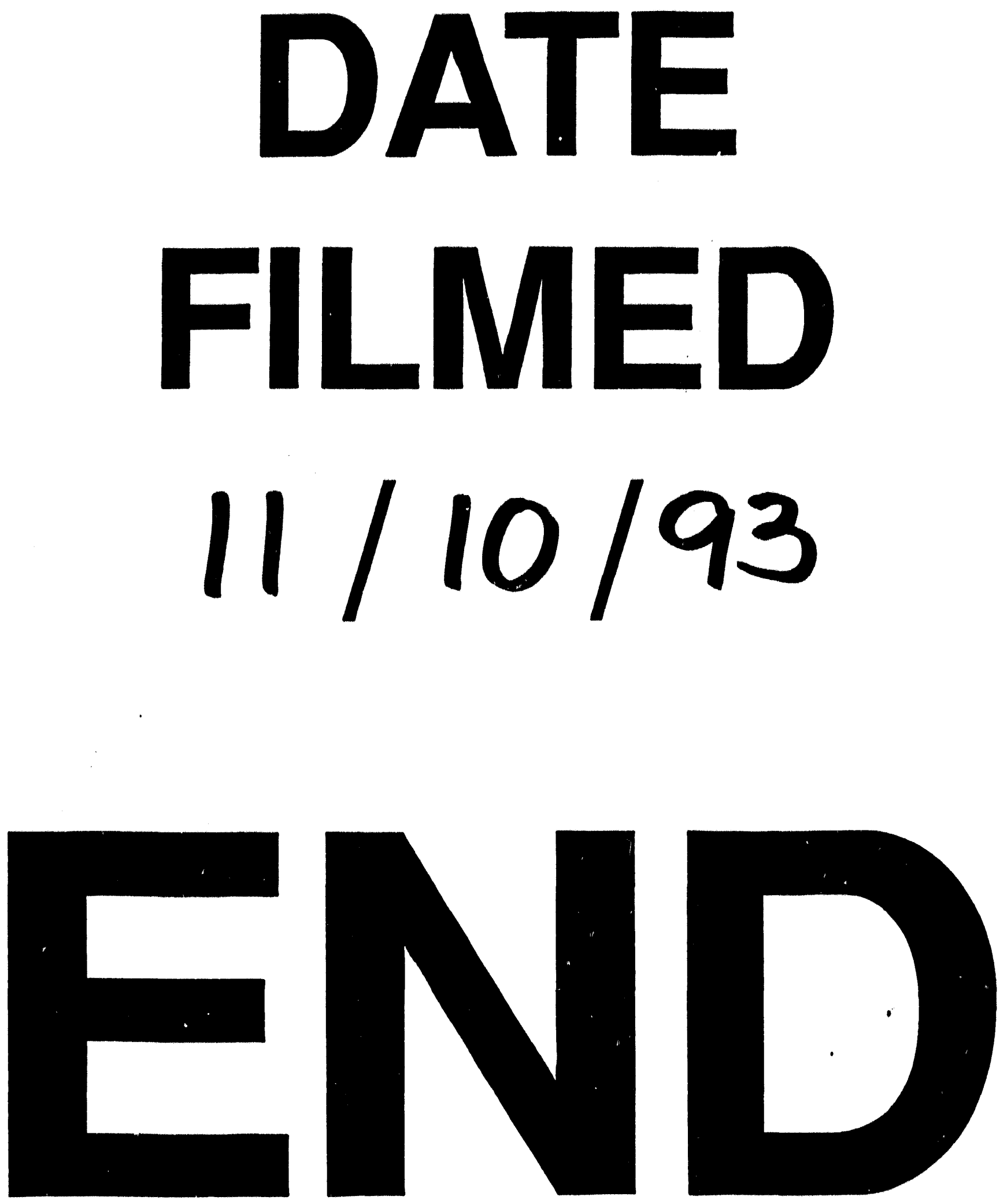